\title{
Early language competence, but not general cognitive ability, predicts children's recognition of emotion from facial and vocal cues
}

\author{
Sarah Griffiths ${ }^{\text {Corresp., } 1}$, Shaun Kok Yew Goh ${ }^{1,2}$, Courtenay Fraiser Norbury ${ }^{1,3}$ \\ 1 Psychology and Language Sciences, University College London, London, United Kingdom \\ 2 Centre for Research in Child Development, Office of Educational Research, National Institute of Education, Nanyang Technological University, Singapore, \\ Singapore \\ 3 Department of Special Needs Education, University of Oslo, Oslo, Norway \\ Corresponding Author: Sarah Griffiths \\ Email address: sarah.griffiths@ucl.ac.uk
}

The ability to accurately identify and label emotions in the self and others is crucial for successful social interactions and good mental health. In the current study we tested the longitudinal relationship between early language skills and recognition of facial and vocal emotion cues in a representative UK population cohort with diverse language and cognitive skills ( $N=369$ ), including a large sample of children that met criteria for Developmental Language Disorder (DLD, $N=97$ ). Language skills, but not non-verbal cognitive ability, at age 5-6 predicted emotion recognition at age 10-12. Children that met the criteria for DLD showed a large deficit in recognition of facial and vocal emotion cues. The results highlight the importance of language in supporting identification of emotions from non-verbal cues. Impairments in emotion identification may be one mechanism by which language disorder in early childhood predisposes children to later adverse social and mental health outcomes. 
1

2

4

5

6 Sarah Griffiths ${ }^{1}$, Shaun Kok Yew Goh ${ }^{1,2}$, Courtenay Frazier Norbury ${ }^{1,3}$ \& the SCALES team

7

81 Psychology and Language Sciences, University College London, London, UK

92 Centre for Research in Child Development, Office of Educational Research, National Institute

10 of Education, Nanyang Technological University, Singapore

113 Department of Special Needs Education, University of Oslo, Oslo, Norway

12

13 Corresponding Author:

14 Sarah Griffiths

15

16
Address: Chandler House, 2 Wakefield Street, London, WC1N 1PF

Email address: sarah.griffiths@ucl.ac.uk 


\section{Abstract}

18 The ability to accurately identify and label emotions in the self and others is crucial for

19 successful social interactions and good mental health. In the current study we tested the

20 longitudinal relationship between early language skills and recognition of facial and vocal

21 emotion cues in a representative UK population cohort with diverse language and cognitive skills

22 ( $\mathrm{n}=369)$, including a large sample of children that met criteria for Developmental Language

23 Disorder (DLD, $\mathrm{n}=97)$. Language skills, but not non-verbal cognitive ability, at age 5-6

24 predicted emotion recognition at age 10-12. Children that met the criteria for DLD showed a

25 large deficit in recognition of facial and vocal emotion cues. The results highlight the importance

26 of language in supporting identification of emotions from non-verbal cues. Impairments in

27 emotion identification may be one mechanism by which language disorder in early childhood

28 predisposes children to later adverse social and mental health outcomes.

29 Keywords: developmental language disorder, emotion recognition, facial expression, vocal expression, longitudinal cohort study, language development. 
32

33

\section{Introduction}

Recognition of emotional cues, such as facial and verbal expressions, is an important social skill. It provides us with information about other people's internal emotional states and helps us to interpret and predict their behaviour. Children have typically acquired the vocabulary for basic emotions by 4-6 years of age (Baron-Cohen, Golan, Wheelwright, Granader, \& Hill, 2010; Ridgeway, Waters, \& Kuczaj, 1985), but accuracy in identifying non-verbal emotional cues continues to improve into late adolescence (Grosbras, Ross, \& Belin, 2018; Herba \& Phillips, 2004; Rodger, Vizioli, Ouyang, \& Caldara, 2015). Accurate emotion identification has been linked to positive outcomes later in development, including academic success (Denham et al., 2012; Izard et al., 2001), social integration (Sette, Spinrad, \& Baumgartner, 2017) and good mental health (Ciarrochi, Scott, Deane, \& Heaven, 2003).

A critical part of learning to identify emotions is developing emotional concepts that align precisely with the emotional concepts held by other people. The Theory of Constructed Emotion (TCE; Gendron \& Barrett, 2018) proposes that language is crucial for acquiring nuanced emotional concepts. Verbal labels provide a framework to organise highly variable input from the environment into coherent emotion concepts (Gendron \& Barrett, 2018; Lindquist, 2017). Critically, the TCE suggests that the role of language in supporting emotion recognition goes beyond acquisition of emotion vocabulary. Precise conceptual alignment is achieved through communication with others. If an individual has less opportunity to learn about emotion concepts through language, their conceptual alignment would be compromised, which would lead to less accurate emotion identification. Previous research has shown that parent-child discourse about emotions predicts children's emotion identification accuracy months later (Dunn, Brown, 
54 Slomkowski, Tesla, \& Youngblade, 1991), consistent with the theory that language aids learning 55 about emotions.

56 In the current study, we test the hypothesis that language supports development of accurate

57 emotion identification by studying a population that has reduced opportunity to learn about

58 emotion concepts through language. Children with Developmental Language Disorder (DLD;

59 previously known as Specific Language Impairment; Bishop, Snowling, Thompson, Greenhalgh,

$60 \&$ The Catalise Consortium, 2017) have difficulties with receptive and/or expressive language

61 that cannot be explained by a sensory deficit or neurological impairments (American Psychiatric

62 Association, 2013). Unlike children with autism spectrum disorders (ASD), children with DLD

63 do not have primary social or emotional difficulties, so any problems with emotion recognition

64 are likely to be a consequence of difficulties acquiring language. If language is necessary for

65 emotion conceptual alignment, children with DLD should have persistent difficulties with

66 emotion understanding, due to reduced opportunity to learn about emotion concepts through

67 language.

68 Children with DLD have been shown to have difficulty with some aspects of emotion

69 understanding; including identifying emotions from hypothetical scenarios (Ford \& Milosky,

70 2003; Spackman, Fujiki, \& Brinton, 2006) and deciding when emotions should be hidden to

71 conform to social display rules (Brinton, Spackman, Fujiki, \& Ricks, 2007). Some studies have

72 found that school-aged children with DLD have difficulty labelling and categorising facial

73 (Bakopoulou \& Dockrell, 2016; Taylor, Maybery, Grayndler, \& Whitehouse, 2015) and verbal

74 (Boucher, Lewis, \& Collis, 2000; Taylor et al., 2015) (Fujiki, Spackman, Brinton, \& Illig, 2008)

75 emotional expressions. However, other studies have not found these differences (Creusere, Alt,

76 \& Plante, 2004; Loukusa, Mäkinen, Kuusikko-Gauffin, Ebeling, \& Moilanen, 2014; Trauner, 
77 Ballantyne, Chase, \& Tallal, 1993) or have observed differences only for some emotions

78 (Spackman, Fujiki, Brinton, Nelson, \& Allen, 2005). This equivocal evidence is likely due to

variable diagnostic criteria, the heterogeneity of the tasks used, and reduced statistical power due to the small sample sizes. The estimated effect size for emotion recognition deficits in ASD, which we would assume to be larger than the size of any deficit in DLD (due to primary challenges in social-emotional processing), is estimated to be 0.41 (Uljarevic \& Hamilton, 2013). Power calculation suggests a sample size of 135 participants in each group is needed to reliably detect an effect of this size (Uljarevic \& Hamilton, 2013). Therefore, much larger studies are required to determine whether children with DLD do have difficulties with emotion identification.

A number of cross-sectional studies in the typically developing population have found associations between language competence and the ability to label and match emotional facial expressions in early childhood (Beck, Kumschick, Eid, \& Klann-Delius, 2012; Pons, Lawson, Harris, \& De Rosnay, 2003; Rosenqvist, Lahti-Nuuttila, Laasonen, \& Korkman, 2014), although other studies have failed to find this relationship (Herba, Landau, Russell, Ecker, \& Phillips, 2006; Herba \& Phillips, 2004). Concurrent relationships between emotion recognition performance and language competence in early childhood may be the result of children not having the vocabulary to meet the language demands in the task. Stronger support for a role of language in refining emotional concepts would come from studies demonstrating a longitudinal relationship between language competence in early childhood and later accuracy in applying labels to emotion cues, at an age when children have acquired basic emotion vocabulary.

In the current study, we use data from a well-characterised longitudinal population cohort that includes children with the full spectrum of language abilities. Children at risk for language 
100 disorder were purposefully oversampled, resulting in a cohort that includes a disproportionately 101 large number of children that meet the criteria for DLD. First, we test the hypothesis that early

102 language competence (age 5-6) is associated with the ability to match facial and vocal emotion

103 cues to basic emotion labels in middle childhood (age 10-12) controlling for children's non-

104 verbal cognitive ability. Second, we test the hypothesis that emotion recognition at age 10-12 is

105 poorer in children that met the criteria for DLD at age 5-6, compared to children with typical

106 language. Finally, we look at error patterns to explore whether children with DLD make similar

107 errors on the emotion recognition tasks to their peers with typical language. The analysis plan for 108 this study was preregistered on the Open Science Framework (osf.io/pwcms).

\section{Materials and Methods}

\section{Sample description}

112 Data are taken from the Surrey Communication and Language in Education Study (SCALES).

113 This study has followed a cohort of children who entered state-maintained schools in the county

114 of Surrey in the United Kingdom in September 2011. Language and communication skills were

115 assessed at school entry via a teacher report questionnaire (Children's Communication Checklist-

116 Short; CCC-S; Norbury et al., 2016). Based on screening, children were classified as having (1)

117 no phrase speech (NPS) (2) high risk for DLD (3) low risk for DLD. Children were classified

118 NPS if their teacher responded 'no' to the question 'is the child combining words into phrases or

119 sentences?' The CCC-S is not applicable for children not speaking in phrases so these children

120 were given the maximum score. The cut-off between high and low risk status was based on age

121 and sex specific cut-offs on the CCC-S derived from the entire screened population $(\mathrm{n}=7267)$

122 (see Norbury et al. 2016 for details). 
123 Stratified random sampling identified a subset of 636 children from the screened population who

124 were invited to take part in direct assessments conducted by trained researchers. Exclusion

125 criteria were (1) attending special schools for children with severe intellectual or physical

126 disability and (2) having English as a second language. All remaining children identified as

127 being NPS $(\mathrm{n}=48)$ were invited, as were 233 low risk and 355 high risk children. Sampling all

128 NPS children and oversampling high-risk children ensured that we had sufficient numbers of

129 children that met the criteria for DLD in the cohort. Five hundred and twenty nine monolingual

130 children were assessed in Year 1, and 384 of these were assessed in Year 6. This final

131 assessment included the emotion recognition tasks. Assessments took around two hours and

132 typically took place at school, although a small number took place during home visits.

133 Language assessments in Year 1 were used to calculate composite scores for expressive

134 language, receptive language, vocabulary, grammar and narrative skills (Norbury et al., 2016).

135 Children were classified as meeting criteria for Developmental Language Disorder (DLD) if they

136 scored -1.5 SD below the mean on at least 2 out of 5 of these composite scores in Year 1 . This is

137 in-line with DSM-5 criteria for Language Disorder that states children must be substantially and

138 quantifiably below age expectations for language across modalities including vocabulary,

139 sentence structure, and discourse (American Psychiatric Association, 2013).

140 Standard scores from block design and matrix reasoning (WPPSI-III; Wechsler, 2003) in Year 1

141 were used to calculate a non-verbal IQ composite by taking the mean of the two scores. This was

142 used to identify children with suspected intellectual disability, defined as a non-verbal IQ

143 composite score of less than -2 SD below the mean. Children that met DLD criteria in Year 1

144 were additionally classified as having DLD with no known associated biomedical condition or

145 DLD with a known associated biomedical condition (hereafter termed LD+). Inclusion criteria 
146 for 'known associated biomedical condition' were (1) intellectual disability based on non-verbal

147 IQ assessments, and/or (2) teacher reported diagnosis of a biomedical condition. Biomedical

148 conditions included; autism, hearing/visual impairment, Down syndrome, epilepsy, neurological

149 impairment, cerebral pals condition including intellectual disability, autism, hearing/visual

150 impairment, Down syndrome, epilepsy, neurological impairment, cerebral palsy,

151 neurofibromatosis and Noonan syndrome (Norbury et al., 2016).

\section{Consent}

153 Consent procedures and study protocol were developed in consultation with Surrey County

154 Council and approved by the Royal Holloway Ethics Committee (where the study started) in

155 Year 1 and the University College London (UCL) Research Ethics Committee in Year 6

156 (9733/002). Informed consent was collected from parents before in-depth assessments in Year 1

157 and Year 6. Informed assent was collected from children prior to the Year 6 assessment.

158 Children were given certificates and small prizes at the end of each assessment session.

\section{Sample size and power calculations}

160 We conducted a priori sensitivity analyses in G-Power based on a sample size estimate of 399

161 participants (assuming a retention rate of 80\% from previous assessment time-point in Year 3).

162 Sensitivity analysis suggested we would have $90 \%$ power to detect small $(r=.15)$ associations

163 between language and emotion recognition accuracy in the whole sample (Cohen, 2013). We

164 also conducted a sensitivity analysis for assessing the group difference between DLD group and

165 the rest of the sample. Assuming equal attrition we estimated that we would have 103 children in

166 Year 6 that had met the DLD criteria in Year 1, including 70 with DLD and no additional

167 diagnosis. Sensitivity analysis suggested that this would provide $90 \%$ power to detect small- 
168 medium group differences $(d=0.34$ for comparison with full DLD group and $d=0.38$ for

169 comparison excluding children with LD with known origin) (Cohen, 2013).

\section{Assessment procedure}

\section{Year 1 Language}

172 In Year 1, children completed 6 tasks to assess receptive and expressive vocabulary, grammar

173 and narrative language skills. Receptive and expressive vocabulary was assessed using the

174 Receptive/Expressive One word Picture Vocabulary Test (R/EOWPVT-4; Martin \& Brownell,

175 2000). These tests have excellent internal consistency for ages 5- to 8-years (Cronbach's $\alpha=.94$ -

176.97 ) and high test-retest reliability (coefficients $=0.97-0.98$ for raw scores). Receptive grammar

177 was assessed using a short form of the Test of Reception of Grammar (TROG-S; Bishop, 2003).

178 The manual reports a split-half reliability for the TROG- 2 of 0.88 , suggesting good internal

179 consistency (Bishop, 2003). Pilot testing demonstrated excellent agreement between short and

180 long forms of $\mathrm{r}(17)=0.88$. Expressive grammar was assessed using the School-Age Sentence

181 Imitation Test (SASIT E32; Marinis, 2011). Expressive narrative skill was assessed using the

182 narrative recall subtest from the Assessment of Comprehension and Expression 6-11 (ACE 6-11;

183 Adams et al., 2001). Cronbach's Alpha of narrative recall for children aged 6- to 11-years is

184 0.73. Finally, receptive narrative skill was assessed using bespoke questions derived from the

185 ACE 6-11 narrative (Adams et al., 2001).

186 Scores for each test were standardised using the LMS method and then averaged to create

187 composite scores for vocabulary (EOWPVT-4 and ROWPVT-4), grammar (TROG-S and

188 SASIT), narrative (ACE recall and comprehension), receptive language (ROWPVT-4, TROG-S

189 and ACE comprehension) and expressive language (EOWPVT-4, SASIT and ACE recall). 
190 Scores on these 5 language composites were used for diagnosing DLD (see participant section

191 above). A total language composite score was created by averaging the standard scores for all 6

192 tests. This language composite was used in the analysis.

193

194

195

196

197

198

199

200

201

202

203

204

205

206

207

208

209

210

211

\section{Year 1 Non-verbal IQ assessment}

In Year 1 children completed two tests of non-verbal IQ (NVIQ); (1) Wechsler Preschool and Primary Scale of Intelligence $3^{\text {rd }}$ edition Block Design and (2) Matrix Reasoning subtests (WPPSI-III; Wechsler, 2003). Standard scores on these two tasks were averaged to create a NVIQ composite score (Norbury et al., 2016).

\section{Year 6 Emotion recognition}

In Year 6 children completed two emotion recognition tasks; one to measure recognition of emotion from faces and one to measure emotion recognition from voices. Each task consisted of 60 trials in which children were presented with photos of faces or recordings of vocal sounds corresponding to one of 6 emotions (happy, sad, angry, surprised, scared and disgusted). For the facial expression task, stimuli were photos of 10 adult actors ( 5 female and 5 male) selected from the Radboud Faces Database (Langner et al., 2010). For the vocal expression task, non-verbal sound stimuli were selected from a validated set of emotional vocal sounds (Sauter, Eisner, Calder, \& Scott, 2010) that have previously been used in research with 6-10 year old children (Sauter, Panattoni, \& Happé, 2013) and adults with autism (Jones et al., 2011). The sounds are made by 4 adult actors ( 2 male and 2 female).

In both tasks, participants were shown a fixation cross for $500 \mathrm{~ms}$, followed by the face stimuli for 2 seconds, or the audio clip accompanied by a cartoon image of a listening man. Participants were then presented with 6 buttons with the emotion labels in a circular formation on the screen. 
212 The labels remained until the participant made a response by pressing the button on the touch

213 screen. The order of the emotion labels on the screen was randomised between participants and

214 tasks, but kept the same between trials for each participant. Total accuracy scores were

215 calculated out of 60 for each task separately.

216 Before completing the task, we checked children's understanding of the 6 emotion words by

217 asking them to read the labels aloud and describe or imitate that emotion. If the child was unable

218 to describe or imitate one or more of the emotions, the assessment was terminated as it was

219 assumed they did not have the basic emotion vocabulary. A very small number of children were

220 not able to read the labels but could describe or imitate the emotion when the word was said

221 aloud. For these children the researcher asked them to give their response verbally during the

222 task and entered their responses for them.

\section{Analysis plan}

\section{Standardisation of scores}

225 Test scores from each of the six language assessments and the two NVIQ assessments in Year 1

226 were standardised using the LMS method (Vamvakas, Norbury, Vitoratou, Gooch, \& Pickles,

227 2019). LMS is a method of standardisation based on the Box-Cox transformation that converts

228 scale raw scores to normality. The resulting scores reflect standardised scores adjusted for age,

229 with a mean of 0 and a standard deviation of 1 . We planned to standardise emotion recognition

230 scores using the same method but this was not necessary as performance was not correlated with

231 age in our sample (faces $r=.05, p=.37$; voices $r=.002, p=.97$ ).

\section{Sampling weights and missing data}


233 Sampling weights were included in all analyses to account for study design and any bias in

234 attrition. This adjustment means that estimates are representative of the screened sample of 6,459

235 monolingual children in state-maintained schools. Sampling weights were produced by

236 multiplying the inverse of the predicted probability of two logistic regression models that predict

237 inclusion in the sample. The first regression model estimates a child's likelihood of being

238 initially invited into the study. This was fitted to the entire population of 6,459 monolingual

239 children in mainstream schools that were screened at school entry. The covariates in this model

240 are those that determined selection into the study due to the stratified sampling method. These

241 are total number of children assessed per school, and whether a child was identified as at risk for

242 DLD based on CCC-S teacher ratings ( $86^{\text {th }}$ centile or above for sex and age group). The second

243 regression model was fitted to the 636 children invited into the study. This model used all

244 available variables to predict retention. This included individual characteristics such as sex,

245 income deprivation score, special education needs, free school meals, English as additional

246 language, Children's Communication Checklist 2 score (D. Bishop, 2003), language in Year 1,

247 season of birth, Strengths and Difficulties Questionnaire (Goodman, 1997)total difficulties score,

248 and school characteristics such as number of pupils on role, percentage of girls, percentage with

249 SEN, and percentage with free school meals. These variables were tested in a stepwise

250 elimination process and included in the model if they predicted inclusion above a cut-off point of 251.2.

\section{Statistical analysis}

253 Statistical analyses were conducted in R version 3.5.3 and M-Plus. Structural Equation Models

254 (SEM) were built under robust maximum likelihood estimator which is robust to deviations from normality. To test the hypothesis that language competence in Year 1 predicts emotion 
256 recognition from faces and voices in Year 6, path analysis was used to model the association

257 between children's composite language scores in Year 1 and their scores on the facial expression

258 and vocal expressions tasks in Year 6. Additionally, because one previous study had suggested

259 that children with DLD may be more impaired in recognition of emotion cues from voices rather

260 than faces (Trauner, Ballantyne, Chase, \& Tallal, 1993) we compared the strength of the

261 pathways between language and performance on the facial expression task and vocal expression

262 task using Wald test of parameter constraints. Finally, we then entered Year 1 NVIQ composite

263 into the model to assess whether language scores continue to predict emotion recognition after

264 accounting for variation in non-verbal cognitive ability.

265 We then compared children in the DLD group to the rest of the sample on total accuracy from

266 the emotion recognition tasks separately. We did not control for NVIQ in this analysis because

267 low NVIQ is not an exclusion criterion for DLD (Bishop, Snowling, Thompson, Greenhalgh, \&

268 The Catalise Consortium, 2017) and language severity is associated with NVIQ (Norbury et al.,

269 2017). This means 'controlling' for group differences in NVIQ would 'control' for relevant and

270 non-random differences between the two groups (Dennis et al. 2009). We conducted this analysis

271 both with and without removing children with additional diagnoses, to determine if there was

272 still a group difference after removing children with co-occurring conditions that have also been

273 associated with problems with emotion recognition (e.g. autism and/or severe intellectual

274 disability).

\section{Results}

276 Of the 384 participants who were seen for assessment in Year 6, 362 (including 67 with DLD

277 and 29 with LD+ additional diagnoses) completed the facial emotion recognition task and 359 
278 (63 with DLD and 27 with LD+ additional diagnoses) completed the vocal emotion recognition 279 task. Three hundred and sixty nine completed at least one task (67 with DLD and 30 with LD+ 280 additional diagnoses) so were included in the analysis. Of the 15 children that did not complete

281 either task, six met criteria for language disorder in Year 1. These children did not complete the 282 task because they did not have the basic emotion vocabulary or were otherwise unable to engage

283 in the task. The other nine children did not have DLD and did not complete the tasks due to

284 technical issues. Table 1 provides descriptive statistics for all variables for the total sample, and 285 DLD, LD+, and typical language groups separately.

286 Attrition was slightly higher than we had anticipated when we conducted our a priori sensitivity analysis. However, our achieved sample size still gave us $90 \%$ power to detect small associations between language and emotion recognition $(r=0.15)$. We also still had $90 \%$ power to detect small-medium size group differences in emotion recognition accuracy between the DLD group and typical language group $(d=0.35)$, even after excluding those with LD+ additional diagnoses $(d=0.41)$

Does early language competence predict later emotion recognition accuracy?

There were moderate prospective relationships between language competence in Year 1 and emotion recognition from vocal expressions $(\beta=.40, \mathrm{~S} . \mathrm{E}=.06,95 \% \mathrm{CI}[.28, .51])$ and facial expressions $(\beta=.42$, S.E $=.06,95 \%$ CI $[.30, .55])$ in Year 6. Wald's test of parameter constraints did not provide evidence for a difference in path strengths between language and emotion recognition from faces and language and emotion recognition from voices $\left(X^{2}(1)=2.51, p=.11\right)$. We had planned on combining the two emotion recognition scores into a single composite score 
300 two outcomes estimated in the model was not sufficient to justify this $(r=.37, \mathrm{~S} . \mathrm{E}=.06,95 \% \mathrm{CI}$

$301[.25, .50])$

302 When NVIQ in Year 1 was entered into the path model as a predictor, the relationships between

303 language and performance on the two emotion recognition tasks remained, and there was no

304 statistical evidence for a prospective relationship between NVIQ and performance on either

305 emotion recognition task (see Fig. 1 for the standardised regression coefficients and confidence

306 intervals for these paths). Table 2 provides the correlation matrix for the variables in the model.

307 Do children with DLD have poorer emotion recognition skills than their peers with typical

308 language?

309 Figure 2 illustrates the distributions of raw accuracy scores on the facial and vocal emotion

310 recognition task for children diagnosed with DLD and children with language in the typical

311 range. Weighted t-tests provided clear statistical evidence for a large group difference in

312 recognition of emotions from faces; $t(360)=4.06, p<.001, d=.90$, and voices $t(353)=4.24, p$

$313<.001, d=0.89$, between these groups. When children with LD+ additional diagnoses were

314 removed, the effect sizes reduced slightly but there was still evidence for a medium-large group

315 difference for recognition of emotion from faces; $t(331)=2.72, p=.007, d=.72$, and voices;

$316 t(326)=2.87, p<.001, d=.78$.

317 Do children with DLD make similar errors in emotion recognition tasks to their peers with 318 typical language?

319 In order to explore possible differences in the kinds of errors made by children with DLD and

320 those without DLD, we created confusion matrices for each task for each group of children (Fig.

321 3.). From these it can be seen that in general the pattern of errors is very similar across the two 
322 groups. The most commonly misidentified emotion in the facial emotion recognition task was

323 disgust in the DLD group and fear in the typical language group and the least commonly

324 misidentified emotion was happiness in both groups. For the vocal emotion recognition task, the

325 most commonly misidentified emotion in both groups was surprise and the least commonly

326 misidentified emotion was happiness in the DLD group and disgust in the typical language

327 group.

\section{Discussion}

329 In the present study we examined the prospective relationship between language competence in early childhood and identification of non-verbal emotion cues in middle childhood in a large population-derived cohort of children with diverse language and cognitive skills. We found evidence for a moderate positive association between language competence at age 5-6 and recognition of facial and vocal emotional cues at age 10-12 supporting our hypothesis that early language skills are positively associated with later emotion recognition ability. The relationship between early language and later emotion recognition held when adjusting for non-verbal cognitive ability, suggesting it is language specifically, rather than cognitive ability more generally, that is associated with later emotion recognition ability.

338 This is the first longitudinal evidence to support the hypothesis from the TCE that language competence plays a role in supporting accurate identification of non-verbal emotion cues

340 (Gendron \& Barrett, 2018). While previous studies have found concurrent associations between 341 language and emotion recognition ability (Beck et al., 2012; Rosenqvist et al., 2014), concurrent 342 associations may be explained by children's language skills limiting their ability to engage with 343 the task. The longitudinal association identified in this study is consistent with the hypothesis 
344 derived from the TCE that having poor language skills has a longer term impact on children's

345 emotion recognition abilities due to children having less refined emotional concepts.

346 We also found that children with DLD have a large deficit in emotion recognition ability. These

347 results help clarify contradictory literature on whether children with DLD have deficits in

348 emotion recognition (Bakopoulou \& Dockrell, 2016; Boucher, Lewis, \& Collis, 2000; Creusere,

349 Alt, \& Plante, 2004; Loukusa, Mäkinen, Kuusikko-Gauffin, Ebeling, \& Moilanen, 2014; Taylor,

350 Maybery, Grayndler, \& Whitehouse, 2015; Trauner et al., 1993). Many of the previous studies

351 have been small ( $\mathrm{n}<20$ children with DLD; Boucher et al., 2000; Loukusa et al., 2014; Taylor et

352 al., 2015; Trauner et al., 1993) and have therefore lacked the statistical power to detect the

353 expected medium-small effect size (Uljarevic \& Hamilton, 2013). Ninety-seven children in our

354 cohort that met the DSM criteria for language disorder when they were 5-6 years old (American

355 Psychiatric Association, 2013), based on rigorous linguistic and cognitive testing, completed at

356 least one emotion recognition task in Year 6, giving us sufficient statistical power. We found

357 strong evidence for a large difference in emotion recognition ability at age 10-12 between those

358 that met the criteria for DLD at age 5-6 compared to those with language in the typical range at

359 age 5-6. When children with other diagnoses that associate with both language and emotion

360 recognition difficulties were excluded from the DLD group (e.g. autism or intellectual

361 disability), the group difference attenuated somewhat, but the statistical evidence for differences

362 between children with DLD and their peers remained strong. This is the largest study to compare

363 children with DLD to typical developing peers on emotion recognition performance, providing

364 the best evidence to date for emotion recognition deficits in DLD.

365 Emotion recognition ability improves with age up until adolescence (Herba \& Phillips, 2004), so

366 we had expected to find an association between emotion recognition performance and age in this 
367 study. The fact that we did not find evidence for age differences in emotion recognition is likely

368 due to the narrow age range in this study (10-12 years) and oversampling of children with

369 suspected DLD. Age related differences within this narrow age range are likely to be small, and

370 therefore obscured by larger individual differences in emotion recognition associated with

371 language disorder.

372 Our emotion recognition task was verbal in the sense that children had to match non-verbal emotion cues to verbal labels. It could therefore be argued that the verbal demands of the task

374 explain the relationship between language competence and emotion recognition performance.

375 However, the labels were basic emotion words that are highly frequent and well within the 376 vocabulary range of children aged 10-12 (Baron-Cohen et al., 2010), even the vast majority of 377 those with DLD. We checked that children understood the emotion words before completing the assessment. A very small number of children with DLD $(n=6)$ lacked the vocabulary to engage

379 in the task, so were not included in the study. Non-verbal tasks, such as a facial expression matching task (Taylor et al., 2015), can be completed using visual features alone, without any comprehension of the underlying emotion and so do not truly test emotion identification. We argue that performance on an emotion labelling task is associated with early language competence not just because it involves a verbal label, but because language is involved in developing nuanced emotion concepts through communication with others throughout childhood (Gendron \& Barrett, 2018).

The ability to recognise and label emotions in the self and others is an important component of social problem solving. The finding that this ability is compromised in DLD may explain why children with DLD are at increased risk of internalising, externalising and ADHD symptoms (Yew \& O'Kearney, 2013). The causal pathway between DLD and poor mental health outcomes 
390 is unclear, but one possibility is that language problems interfere with aspects of social-

391 emotional processing (such as emotion concept development), which in turn leads to negative

392 social, emotional and mental health outcomes. Im-Bolter, Cohen, and Farnia (2013) found that

393 adolescents referred to mental health services had poorer structural and figural language than

394 peers recruited from the community and were poorer at social problem solving. The findings in

395 the current study raise the possibility that emotion identification may be one pathway in which

396 poor language in early childhood compromises social functioning and mental health in children

397 with and without DLD.

398 There are a number of possible pathways from poor early language to later emotion recognition 399 difficulties, which should be explored in future studies. First, we assume that children with DLD 400 have less opportunity to learn about emotion through communication with caregivers but we 401 have not directly tested this. Future studies should explore the quality of parent-child discourse 402 about emotions and test whether this is associated with children's emotion recognition. A second 403 promising avenue for future research is the role of alexithymia in explaining the association 404 between language and emotion recognition. Alexithymia describes difficulties identifying and 405 reporting one's own emotional state. It has been proposed recently that language disorder is one 406 route to alexithymia (Hobson, Brewer, Catmur, \& Bird, 2019) and alexithymia is associated with 407 impairments in recognising non-verbal emotional cues in others such as facial expressions 408 (Cook, Brewer, Shah, \& Bird, 2013). Future studies should include measures of alexithymia to 409 determine to what extent this trait explains the association between early language and later 410 emotion recognition ability.

411 Although our findings are consistent with a causal relationship between language competence 412 and emotion recognition, they cannot provide proof of causality. One way to investigate whether 
413 this relationship is truly causal would be to test whether interventions aimed at improving

414 language have positive, cascading effects on emotion recognition skills later in development.

415 Interventions that focus specifically on language skills directly related to emotion understanding

416 are more likely to transfer on emotion recognition than general language interventions, which

417 may be too distal for transfer to occur. To date there has been one preliminary study $(\mathrm{n}=208)$

418 investigating whether a nine-week intervention focusing specifically on improving language

419 related to emotion through storybooks improves other emotional skills in typically developing 7-

4209 year old children. The intervention group showed improvements in emotional vocabulary,

421 emotion knowledge and recognition of masked emotions from vignettes compared to a

422 'treatment as usual' control group straight after the intervention $(\beta=1.05-1.32$; Kumschick et al.,

423 2014). Future research is needed to determine whether interventions focused on language for

424 emotion can improve emotion recognition skills in children with DLD.

\section{Conclusions}

426 In conclusion, this study provides the first longitudinal evidence that early language skills

427 specifically predict later emotion recognition from both facial and vocal cues. These findings

428 support the hypothesis that language plays a role in supporting emotion identification (Barrett,

429 Lindquist, \& Gendron, 2007; Gendron \& Barrett, 2018; Lindquist, 2017). Children with DLD are

430 therefore especially vulnerable to difficulties recognising their own and others' emotional states.

431 We propose that this deficit may be one causal mechanism that underpins the reported

432 relationship between early language skills and later adverse mental health. 


\section{Acknowledgements}

435 We thank Surrey County Council for facilitating the data collection process and the children, 436 parents, schools and teachers for taking part in the study. We also thank the other members of the 437 SCALES team: Debbie Gooch, Gillian Baird, Tony Charman, Andrew Pickles and Emily 438 Simonoff for their advice. Finally, we thank Dorothy Bishop for permission to develop the 439 Children's Communication Checklist-Short and allowing us access to the standardization data.

440 The views expressed in this article are those of the authors and not necessarily those of the 441 Wellcome Trust, the ESRC, the British Academy or Surrey County Council.

\section{Open Practices Statement}

443 The hypotheses and analysis plan for this study was preregistered on the Open Science

444 Framework (osf.io/pwcms). Twelve participant's parents did not consent to Open Data sharing 445 so we are unable to share the full dataset. However, our code and a dataset with these 12 446 participants removed is available on the OSF page for this study. 
447

448

449

450

451

452

453

454

455

456

457

458

459

460

461

462

463

464

465

466

467

468

469

470

471

472

473

474

475

476

477

478

479

480

481

482

483

484

485

486

487

488

489

490

491

492

493

494

495

496

497

498

499

500

501

\section{References}

American Psychiatric Association. (2013). Diagnostic and statistical manual of mental disorders: DSM-5 (5th ed.). Arlington, VA: American Psychiatric Publishing.

Bakopoulou, I., \& Dockrell, J. E. (2016). The role of social cognition and prosocial behaviour in relation to the socio-emotional functioning of primary aged children with specific language impairment. Research in Developmental Disabilities, 49-50, 354-370. doi:https://doi.org/10.1016/j.ridd.2015.12.013

Baron-Cohen, S., Golan, O., Wheelwright, S., Granader, Y., \& Hill, J. (2010). Emotion word comprehension from 4 to 16 years old: a developmental survey. Frontiers in evolutionary neuroscience, 2, 109-109. doi:10.3389/fnevo.2010.00109

Barrett, L. F., Lindquist, K. A., \& Gendron, M. (2007). Language as context for the perception of emotion. Trends in cognitive sciences, 11(8), 327-332. doi:https://doi.org/10.1016/j.tics.2007.06.003

Beck, L., Kumschick, I. R., Eid, M., \& Klann-Delius, G. (2012). Relationship between language competence and emotional competence in middle childhood. Emotion, 12(3), 503-514. doi:10.1037/a0026320

Bishop, D. (2003). The Children's Communication Checklist version 2 (CCC-2) Psychological Corporation. London, England.

Bishop, D. V. M. (2003). TROG-2 test for reception of grammar-2: London: Harcourt.

Bishop, D. V. M., Snowling, M. J., Thompson, P. A., Greenhalgh, T., \& The Catalise Consortium. (2017). Phase 2 of CATALISE: a multinational and multidisciplinary Delphi consensus study of problems with language development: Terminology. Journal of Child Psychology and Psychiatry, 58(10), 1068-1080. doi:10.1111/jcpp.12721

Boucher, J., Lewis, V., \& Collis, G. M. (2000). Voice Processing Abilities in Children with Autism, Children with Specific Language Impairments, and Young Typically Developing Children. Journal of Child Psychology and Psychiatry, 41(7), 847-857. doi:10.1111/1469-7610.00672

Brinton, B., Spackman, M. P., Fujiki, M., \& Ricks, J. (2007). What Should Chris Say? The Ability of Children With Specific Language Impairment to Recognize the Need to Dissemble Emotions in Social Situations. Journal of Speech, Language, and Hearing Research, 50(3), 798-811. doi:doi:10.1044/1092-4388(2007/055)

Ciarrochi, J., Scott, G., Deane, F. P., \& Heaven, P. C. L. (2003). Relations between social and emotional competence and mental health: a construct validation study. Personality and Individual Differences, 35(8), 1947-1963. doi:https://doi.org/10.1016/S0191-8869(03)00043-6

Cohen, J. (2013). Statistical power analysis for the behavioral sciences: Routledge.

Cook, R., Brewer, R., Shah, P., \& Bird, G. (2013). Alexithymia, Not Autism, Predicts Poor Recognition of Emotional Facial Expressions. Psychol Sci, 24(5), 723-732. doi:10.1177/0956797612463582

Creusere, M., Alt, M., \& Plante, E. (2004). Recognition of vocal and facial cues to affect in languageimpaired and normally-developing preschoolers. Journal of Communication Disorders, 37(1), 520. doi:https://doi.org/10.1016/S0021-9924(03)00036-4

Denham, S. A., Bassett, H. H., Way, E., Mincic, M., Zinsser, K., \& Graling, K. (2012). Preschoolers' emotion knowledge: self-regulatory foundations, and predictions of early school success. Cogn Emot, 26(4), 667-679. doi:10.1080/02699931.2011.602049

Dunn, J., Brown, J., Slomkowski, C., Tesla, C., \& Youngblade, L. (1991). Young children's understanding of other people's feelings and beliefs: individual differences and their antecedents. Child Dev, 62(6), 1352-1366.

Ford, J. A., \& Milosky, L. M. (2003). Inferring emotional reactions in social situations: differences in children with language impairment. J Speech Lang Hear Res, 46(1), 21-30. doi:10.1044/10924388(2003/002)

Fujiki, M., Spackman, M. P., Brinton, B., \& Illig, T. (2008). Ability of children with language impairment to understand emotion conveyed by prosody in a narrative passage. International Journal of Language \& Communication Disorders, 43(3), 330-345. doi:10.1080/13682820701507377

Gendron, M., \& Barrett, L. F. (2018). Emotion Perception as Conceptual Synchrony. Emotion Review, 10(2), 101-110. doi:10.1177/1754073917705717

Goodman, R. (1997). The Strengths and Difficulties Questionnaire: a research note. Journal of Child Psychology and Psychiatry, 38(5), 581-586. 
502

503

504

505

506

507

508

509

510

511

512

513

514

515

516

517

518

519

520

521

522

523

524

525

526

527

528

529

530

531

532

533

534

535

536

537

538

539

540

541

542

543

544

545

546

547

548

549

550

551

552

553

554

555

Grosbras, M.-H., Ross, P. D., \& Belin, P. (2018). Categorical emotion recognition from voice improves during childhood and adolescence. Scientific Reports, 8(1), 14791. doi:10.1038/s41598-01832868-3

Herba, C., Landau, S., Russell, T., Ecker, C., \& Phillips, M. (2006). The development of emotionprocessing in children: effects of age, emotion, and intensity. Journal of Child Psychology and Psychiatry, 47(11), 1098-1106. doi:10.1111/j.1469-7610.2006.01652.x

Herba, C., \& Phillips, M. (2004). Annotation: Development of facial expression recognition from childhood to adolescence: behavioural and neurological perspectives. Journal of Child Psychology and Psychiatry, 45(7), 1185-1198. doi:10.1111/j.1469-7610.2004.00316.x

Hobson, H., Brewer, R., Catmur, C., \& Bird, G. (2019). The Role of Language in Alexithymia: Moving Towards a Multiroute Model of Alexithymia. Emotion Review, 11(3), 247-261. doi:10.1177/1754073919838528

Im-Bolter, N., Cohen, N. J., \& Farnia, F. (2013). I thought we were good: social cognition, figurative language, and adolescent psychopathology. J Child Psychol Psychiatry, 54(7), 724-732. doi:10.1111/jcpp.12067

Izard, C., Fine, S., Schultz, D., Mostow, A., Ackerman, B., \& Youngstrom, E. (2001). Emotion knowledge as a predictor of social behavior and academic competence in children at risk. Psychol Sci, 12(1), 18-23. doi:10.1111/1467-9280.00304

Jones, C. R., Pickles, A., Falcaro, M., Marsden, A. J., Happe, F., Scott, S. K., . . Charman, T. (2011). A multimodal approach to emotion recognition ability in autism spectrum disorders. $J$ Child Psychol Psychiatry, 52(3), 275-285. doi:10.1111/j.1469-7610.2010.02328.x

Kumschick, I. R., Beck, L., Eid, M., Witte, G., Klann-Delius, G., Heuser, I., . . Menninghaus, W. (2014). READING and FEELING: the effects of a literature-based intervention designed to increase emotional competence in second and third graders. Frontiers in Psychology, 5(1448). doi:10.3389/fpsyg.2014.01448

Langner, O., Dotsch, R., Bijlstra, G., Wigboldus, D. H. J., Hawk, S. T., \& van Knippenberg, A. (2010). Presentation and validation of the Radboud Faces Database. Cognition and Emotion, 24(8), 1377-1388. doi:10.1080/02699930903485076

Lindquist, K. A. (2017). The role of language in emotion: existing evidence and future directions. Current Opinion in Psychology, 17, 135-139. doi:https://doi.org/10.1016/j.copsyc.2017.07.006

Loukusa, S., Mäkinen, L., Kuusikko-Gauffin, S., Ebeling, H., \& Moilanen, I. (2014). Theory of mind and emotion recognition skills in children with specific language impairment, autism spectrum disorder and typical development: group differences and connection to knowledge of grammatical morphology, word-finding abilities and verbal working memory. International Journal of Language \& Communication Disorders, 49(4), 498-507. doi:10.1111/1460-6984.12091

Marinis, T., Chiat, S., Armon-Lotem, S., Piper, J., \& Roy, P. . (2011). School-age sentence imitation test-E32. . http://www.city.ac.uk/health/research/centre-for-language-communication-sciencesresearch/veps-very-early-processing-skills/veps-assessments [last accessed 27 April 2016].

Norbury, C. F., Vamvakas, G., Gooch, D., Baird, G., Charman, T., Simonoff, E., \& Pickles, A. (2017). Language growth in children with heterogeneous language disorders: a population study. Journal of Child Psychology and Psychiatry, 58(10), 1092-1105. doi:10.1111/jcpp.12793

Pons, F., Lawson, J., Harris, P. L., \& De Rosnay, M. (2003). Individual differences in children's emotion understanding: Effects of age and language. Scandinavian Journal of Psychology, 44(4), 347353. doi:10.1111/1467-9450.00354

Ridgeway, D., Waters, E., \& Kuczaj, S. A. (1985). Acquisition of emotion-descriptive language: Receptive and productive vocabulary norms for ages 18 months to 6 years. Dev Psychol, 21(5), 901.

Rodger, H., Vizioli, L., Ouyang, X., \& Caldara, R. (2015). Mapping the development of facial expression recognition. Developmental Science, 18(6), 926-939. doi:10.1111/desc.12281

Rosenqvist, J., Lahti-Nuuttila, P., Laasonen, M., \& Korkman, M. (2014). Preschoolers' recognition of emotional expressions: Relationships with other neurocognitive capacities. Child Neuropsychology, 20(3), 281-302. doi:10.1080/09297049.2013.778235

Sauter, D. A., Eisner, F., Calder, A. J., \& Scott, S. K. (2010). Perceptual Cues in Nonverbal Vocal Expressions of Emotion. Quarterly Journal of Experimental Psychology, 63(11), 2251-2272. doi:10.1080/17470211003721642

Peer) reviewing PDF | (2020:01:45198:1:1:NEW 9 Apr 2020) 
556

557

558

559

560

561

562

563

564

565

566

567

568

569

570

571

572

573

574

575

576

577

578

579

580

581

582

583

584
Sauter, D. A., Panattoni, C., \& Happé, F. (2013). Children's recognition of emotions from vocal cues. British Journal of Developmental Psychology, 31(1), 97-113. doi:10.1111/j.2044835X.2012.02081.x

Sette, S., Spinrad, T. L., \& Baumgartner, E. (2017). The Relations of Preschool Children's Emotion Knowledge and Socially Appropriate Behaviors to Peer Likability. Int J Behav Dev, 41(4), 532541. doi:10.1177/0165025416645667

Spackman, M. P., Fujiki, M., \& Brinton, B. (2006). Understanding emotions in context: the effects of language impairment on children's ability to infer emotional reactions. International Journal of Language \& Communication Disorders, 41(2), 173-188. doi:10.1080/13682820500224091

Spackman, M. P., Fujiki, M., Brinton, B., Nelson, D., \& Allen, J. (2005). The Ability of Children With Language Impairment to Recognize Emotion Conveyed by Facial Expression and Music. Communication Disorders Quarterly, 26(3), 131-143. doi:10.1177/15257401050260030201

Taylor, L. J., Maybery, M. T., Grayndler, L., \& Whitehouse, A. J. O. (2015). Evidence for shared deficits in identifying emotions from faces and from voices in autism spectrum disorders and specific language impairment. International Journal of Language \& Communication Disorders, 50(4), 452466. doi:10.1111/1460-6984.12146

Trauner, D. A., Ballantyne, A., Chase, C., \& Tallal, P. (1993). Comprehension and expression of affect in language-impaired children. Journal of Psycholinguistic Research, 22(4), 445-452. doi:10.1007/bf01074346

Uljarevic, M., \& Hamilton, A. (2013). Recognition of Emotions in Autism: A Formal Meta-Analysis. Journal of Autism and Developmental Disorders, 43(7), 1517-1526. doi:10.1007/s10803-012-1695-5

Vamvakas, G., Norbury, C. F., Vitoratou, S., Gooch, D., \& Pickles, A. (2019). Standardizing test scores for a target population: The LMS method illustrated using language measures from the SCALES project. PLoS ONE, 14(3), e0213492. doi:10.1371/journal.pone.0213492

Yew, S. G. K., \& O'Kearney, R. (2013). Emotional and behavioural outcomes later in childhood and adolescence for children with specific language impairments: meta-analyses of controlled prospective studies. Journal of Child Psychology and Psychiatry, 54(5), 516-524. doi:doi:10.1111/jcpp.12009 
Figure 1

Path model showing prospective relationships from language (Lang) and non-verbal IQ (NVIQ) in Year 1 to emotion recognition from faces (ER faces) and voices (ER voices) in Year 6 . Significant paths are solid lines while insignificant paths are dashed line

Significant paths are solid lines while insignificant paths are dashed line.

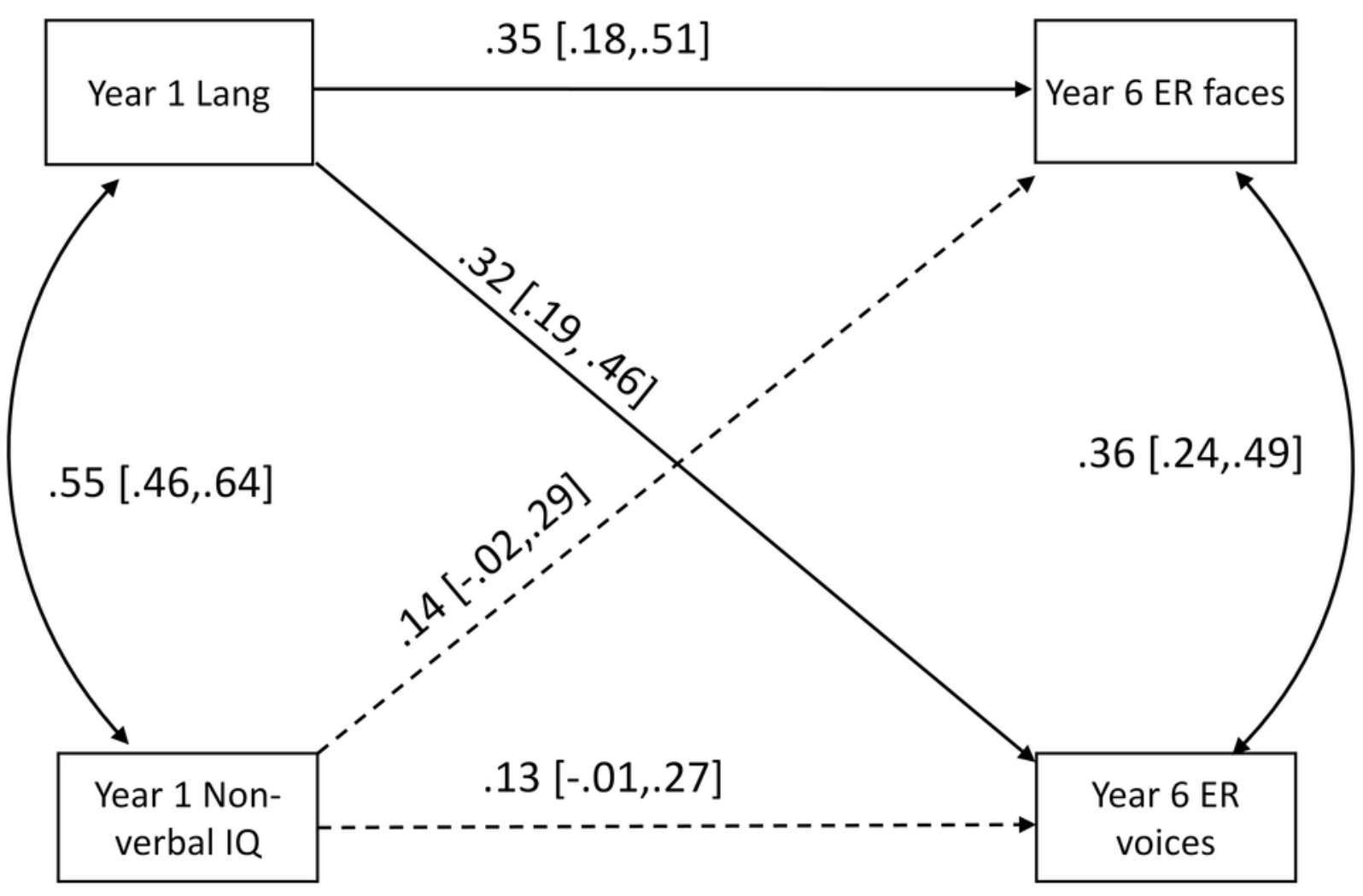


Figure 2

Pirate plot showing distribution of total scores on $(A)$ the vocal emotion recognition task and $(B)$ the facial emotion recognition task for group with DLD and the typically language group.
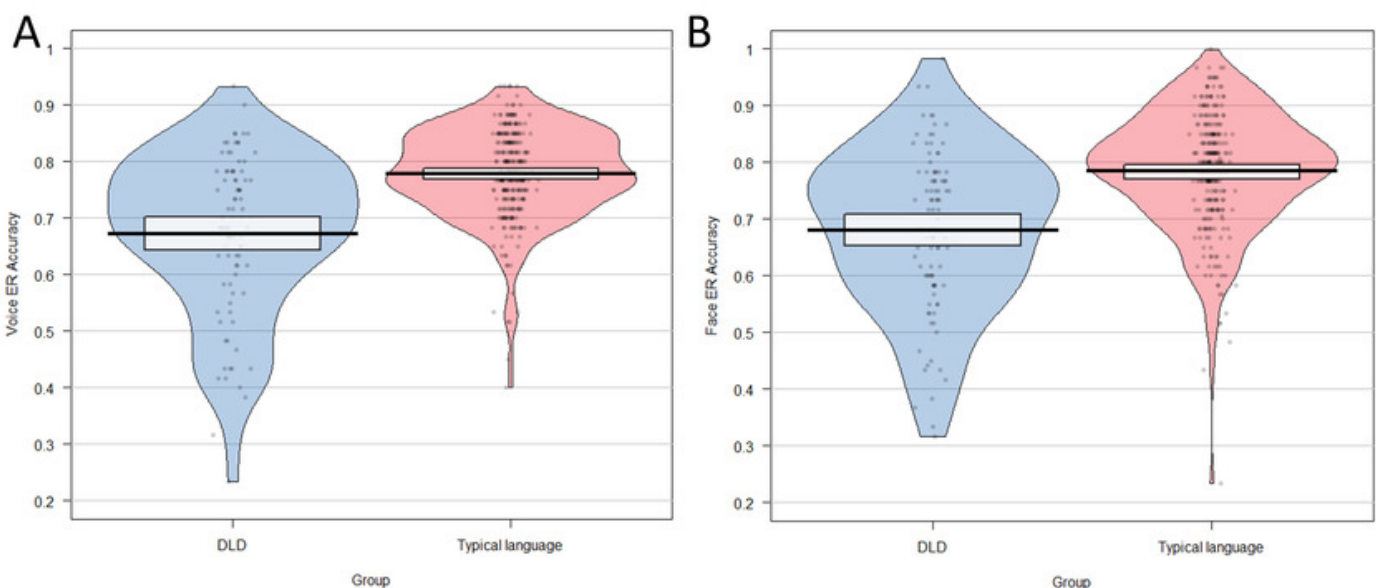
Figure 3

Confusion matrices showing proportion of responses in each category for each presented emotion separately by language group for (a) faces and (b) voices. 


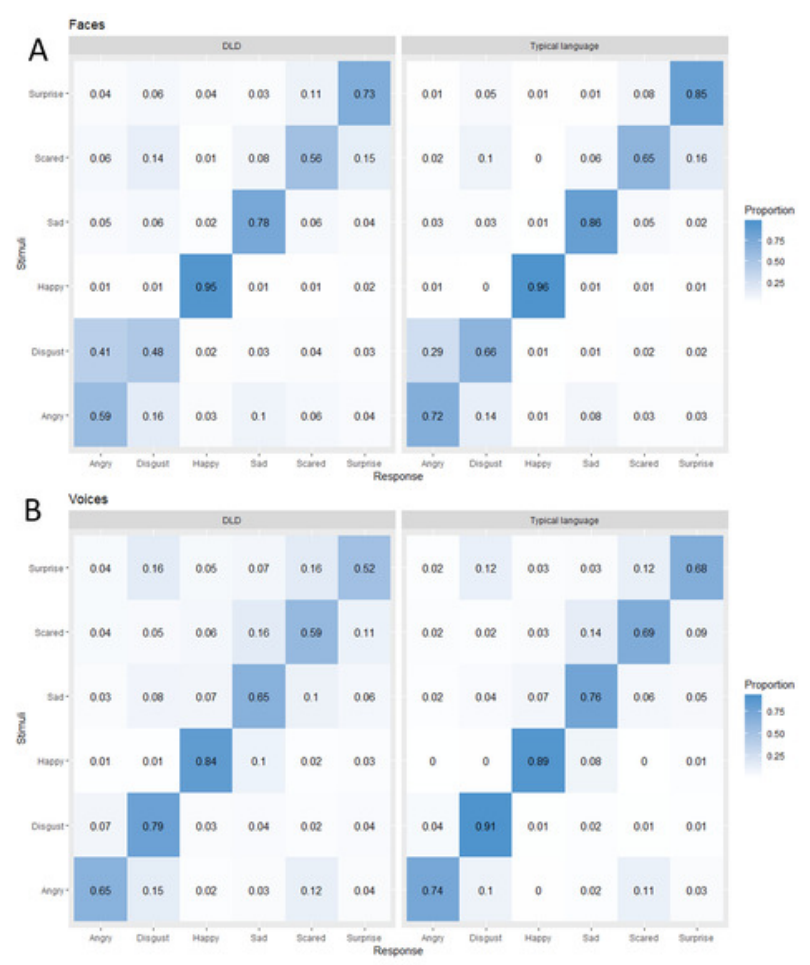




\section{Table 1 (on next page)}

Descriptive statistics for the full sample and each language group separately.

The language composite score is the average of standard scores from the six language assessments. The NVIQ composite is the averaged of standard scores from the two nonverbal IQ assessments. Emotion recognition scores are raw total accuracy scores on each task. 
1 Table 1:

2 Descriptive statistics for the full sample and each language group separately.

3 The language composite score is the average of standard scores from the six language

4 assessments. The NVIQ composite is the averaged of standard scores from the two non-verbal

5 IQ assessments. Emotion recognition scores are raw total accuracy scores on each task.

\begin{tabular}{lcccc} 
& $\begin{array}{c}\text { Full sample } \\
\mathrm{n}=369\end{array}$ & $\begin{array}{c}\text { Typical } \\
\text { language } \\
\mathrm{n}=272\end{array}$ & $\begin{array}{c}\text { DLD } \\
\mathrm{n}=67\end{array}$ & $\begin{array}{c}\text { LD }+ \\
\mathrm{n}=30\end{array}$ \\
\hline Mean $(S D)$ & Mean $(S D)$ & Mean $(S D)$ & Mean (SD) \\
\hline Age Year 1 (Years) & $5.97(0.39)$ & $5.97(0.40)$ & $5.90(0.35)$ & $6.13(0.34)$ \\
Age Year 6 (Years) & $11.16(0.34)$ & $11.16(0.34)$ & $11.12(0.36)$ & $11.24(0.31)$ \\
Male n (\%) & $185(50 \%)$ & $129(47 \%)$ & $37(55 \%)$ & $19(63 \%)$ \\
Language composite Year 1 & $-0.59(1.06)$ & $-0.09(0.85)$ & $-1.78(0.49)$ & $-2.50(0.67)$ \\
NVIQ composite Year 1 & $-0.39(1.07)$ & $-0.13(0.98)$ & $-0.83(0.65)$ & $-1.81(1.19)$ \\
ER faces Year 6 & $0.76(0.12)$ & $0.79(0.10)$ & $0.71(0.14)$ & $0.61(0.13)$ \\
ER voices Year 6 & $0.75(0.11)$ & $0.78(0.08)$ & $0.71(0.12)$ & $0.59(0.16)$
\end{tabular}

6

$7 \quad \mathrm{NVIQ}=$ non-verbal IQ; ER = Emotion recognition

8 a Based on 362 total, 272 TL, 67 DLD, 29 LD+

9 b Based on 359 total, 278 TL, 63 DLD, 30 LD+ 


\section{Table 2 (on next page)}

Correlations between variables included in the path model. The language composite score is the average of standard scores from the 6 language assessments. The NVIQ composite is the averaged of standard scores from the 2 non-verbal IQ assessments. Emotion

The language composite score is the average of standard scores from the 6 language assessments. The NVIQ composite is the averaged of standard scores from the 2 non-verbal IQ assessments. Emotion recognition scores are raw total accuracy scores on each task. 
1 Table 2:

2 Correlations between variables included in the path model.

3 The language composite score is the average of standard scores from the 6 language assessments.

4 The NVIQ composite is the averaged of standard scores from the 2 non-verbal IQ assessments.

5 Emotion recognition scores are raw total accuracy scores on each task.

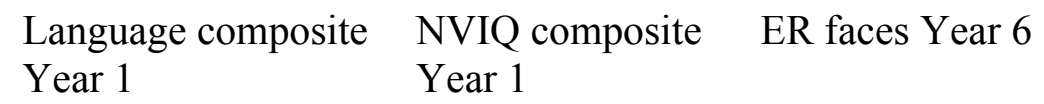

NVIQ composite Year $1 \quad 0.55$

ER faces Year $6 \quad 0.42 \quad 0.33$

$\begin{array}{llll}\text { ER voices Year } 6 & 0.40 & 0.31 & 0.48\end{array}$

6

$7 \quad \mathrm{NVIQ}=$ non-verbal IQ; ER = Emotion recognition 